\title{
Probe into Measures to Improve the Accuracy of Coal Mine Geological Survey
}

\section{Pengfei Zhang}

Shanxi Jincheng Anthracite Mining Group Co., Ltd., Jincheng, Shanxi, 048000, China

\begin{abstract}
In the process of China's development, various resources need to be used effectively. Among them, coal resources are one of the important basic guarantees in people's life and production. The continuous use of coal resources in development has made mining more and more difficult, and put forward higher challenges to the mining process and the geological survey process. During the construction of coal mining projects, it is necessary to carry out effective measurement data, accurately improve the underground construction situation of the entire coal mine, make detailed data sorting of underground mines, and make correct judgments. Based on the actual situation, the thesis improves the accuracy in the process of coal mine geological survey, analyzes the related reasons, and adopts scientific measures to improve the maximization of coal mining.
\end{abstract}

\section{Keywords}

coal mine; geological survey; accuracy; improvement

\section{煤矿地质测量精度提升措施探究}

\section{张鹏飞 \\ 山西晋城无烟煤矿业集团有限责任公司，中国・山西 晋城 048000}

\section{摘 要}

在中国发展过程中, 需要对各种资源进行有效利用。其中, 煤矿资源是人们生活生产中重要的基础保障之一。对于发展中不 断利用煤矿资源, 导致开采难度越来越大, 对挖掘的过程与地质测量过程提出了更高的挑战。在煤炭挖掘工程施工期间, 要 进行有效的测量数据, 精准地提升整个煤矿地下施工情况, 对地下矿井做出详细的数据整理, 做出正确的判断。论文从实际 出发, 在煤矿地质测量过程中提高精准度, 并对相关原因进行分析, 通过科学的措施, 提高煤矿采集的最大化。

\section{关键词}

煤矿; 地质测量; 精度; 提升

\section{1 引言}

近年来，中国煤矿事业发展速度很快，为有效的全面保 证煤矿事业进行长期稳定的发展, 其中测量工作的重要性便 不言而喻。煤矿地质测量工作是整个生产作业的基础, 它的 作用不言而喻, 不可估量。众所周知, 测量是一项容不得半 点马虎的工作, 尤其是煤矿地质测量这么重要的工作, 更需 要相关工作人员自身保持严谨认真的态度, 踏实稳定的作风。 如果拥有马虎大意等现象的发生, 很容易因为一点小失误从 而全面影响整个工作的展开, 甚至给工人带来不可挽回的损 失, 所以严谨的工作态度是很重要的。

\section{2 煤矿测量工作的重要性分析}

煤矿测量工作在煤矿开采当中非常重要, 在开展测量工
作当中如果发生任何施工，都有可能导致大的事故发生，这 些事故会对工作人员的生命财产造成非常大的安全隐患。煤 矿生产大部分都是在深至几百米的矿井下面，很多时间久的 煤矿经过长时间的开采，会导致矿井内部的管道错综复杂。 因为煤矿的测量准确性对对煤矿的生产安全十分重要的作用, 在对其开展测量的过程当中，一定要确保生产安全，防治安 全问题的发生。要强化工作人员的安全认知，通过专业理论 知识和实践经验解决在煤矿测量中出现的问题。

\section{3 煤矿地质测量精度影响要素}

由于中国经济的发展，对其精度要求不断提高。同 时在 精度提高后，也可以保障整个生产过程中安全有效 的运行。 煤矿检测过程中, 涉及众多的环节, 如施工 测量、数据采集、 
数据运算以及绘制测量图纸等, 根据 测量的数据做好整理工 作, 加强各个环节的有效连接, 若某一环节出现了问题, 则 会导致后续多个环节造成严 重影响, 最终精准度的产生较大 差异, 然而从影响测量 精准的因素分析, 从以下几个方面进 行人手。

\section{1 测量设备的选取及其精准度}

在地质测量工作开展过程中, 要依靠专业的设备, 对其 作出精准的测量数值, 设备是测量精准度的基础, 结合测绘 数据的精准度, 能够科学的分析出地质的实际 情况。在测量 前要对测量设备做好校对工作, 避免由于设备精准度不足, 对测量结果产生偏差。必须综合测量精准度需求与工作进行 环境的情况来选择测绘的仪器, 另外对选取的仪器实施细致 的检查和校对, 从而有效规避因为仪器的不合格对测量的结 果造成干扰。

\section{2 地质测点的篮选}

受地质开采的影响, 当井下水准点、三角点等地质测点, 在测量过程中会发生变动, 参数位置数据也会产生变化, 对 后期测量数据造成相应的影响。在地质数据 检测过程中, 根 据数据进行分析, 降低测量结果的差异。因此在开展过程中, 对地质测量工作前要进行测量点的校正, 针对测量点进行筛 选以及确定保证测量工作的有效进行，保证在施工期间不会 发生检测点的位置变动。

\section{3 工作人员的技能水平}

在进行任何创造生产任务时都离不开重要的组成部分, 在进行矿下地质测量精准度时更是离不开相关专业技能工作 者, 因此, 在进行测量工作中, 专业技术工作者的自身素质 和习性都会对测量的结果有着重要的影响, 严重时会由于部 分工作者的操作错误, 致使测量的结果产生误差。

\section{4 煤矿地质测量精度的提升措施}

\section{1 完善前期准备工作}

做好前期的准备工作是确保测量精确度的重要前提, 相 关人员应该做好前期工作, 这样才能减少测量的误差产生。 具体可以分为以下几点: (1) 在测量前先准备好相应的设备, 而且还要将设备的参数设置好, 做好相应的校队工作, 这样 能够减少在工作中产生的数据差距, 尽可能的减少设备造成 的误差。（2）在开展相关工作前, 应该让专业人员对现场进
行实地考察, 同时根据地质和环境来对其做出一个评估, 这 样能够找到更好的处理措施，降低在测量过程中其他问题的 产生。（3）该项工作都有具有较好的审核体系，相关的工作 人员可以根据当前的实际情况不断去完善该体系, 这样能够 对数据进行全面的审核, 保证数据的有效性, 从而减少误差 的产生。

\section{2 核对测量原始数数据}

在实际的测量工作中, 相关工作人员想要保证数据的有 效性, 则需要一个参考数据作为对比, 为此, 保证初始数据 的准确性有十分重要的作用, 其能够作为当前矿井测量的基 础内容。在开展相关工作时, 工作人员可以利用初始数据作 为参数对测量的数据进行对比, 利用初始数据对其进行分析, 能够很快的发现存在的异常问题, 从而找到存在异常的原因, 并利用有效的处理措施进行处理, 而且还可以对此次的问题 加以记录,一旦再产生该类型问题时, 可以更好做出相应处理。 另外, 对矿井进行测绘时的初始数值过多, 为了确保运算活 动能够正常运行, 在进行核算活动以前必须创建比较细致的 初核与复核程序, 保证不会发生初始数值丢失的状况, 另外 还能够提高初始数值的准确程度。矿井地质测量工作内容比 较多, 而且其结构比较繁琐, 相关内容都是一环与一环相接, 如果在展开工作的过程中出现失误或是遗漏等现象就会造成 较大的误差, 为此, 相关人员应该在展开工作的过程中安排 好相关内容, 保证流程能够顺利展开, 并不遗漏数据信息。

\section{3 加大对专业测量人员的培训力度}

应该将日常培训加以重视, 提升对测绘专业工作者的管 控。矿产地质测绘的根本就在于拥有专业的测绘工作者, 相 关工作者的技术水准与素养的好坏, 对以后的测绘成果直接 起着重要的作用。所以, 想要提升测绘的准确程度, 矿产公 司必须要重点考虑测绘工作者的培训技能, 提升有关治理。 在学习的方法上比较多样化, 例如, 将相关技术工作者派去 其他矿上进行学习, 进而提高测绘工作者的工作技术能力。 另外还可以重点考虑测绘工作者的考核检测, 促使专业工作 者自主的提升自身的水平和素养。需要规范性的对有关工作 进行安排, 以最大程度规避认为因素带来的测绘误差。

\section{4 准确绘制设计图纸}

在开展相关工作前, 需要对该次工程进行绘图, 并通过 绘图内容来实施方案, 绘图工作是开展该项工作的基础内容。 
在开展相关工作前, 需要专业的工作人员对其进行绘图, 并 根据实际情况作出较好的调整, 这样有利于后续工作的展开, 同时还能获得较为准确的数据信息，将工作内容不断优化。 通常情况下, 绘图内容的精准度越高, 内容越详细, 工作人 员工作起来更加简便, 工作人员可以通过绘图内容找到地下 反应的实际情况, 进而找到较好的处理措施, 保证数据的精 确性。通常情况下, 在绘图过程中应该注意一下几点问题:

（1）绘图人员应该对实地进行考察, 根据地质和气候变化来 进行不断调整, 从而给工作人员提供一个良好的信息, 提高 其工作效率。（2）绘图的过程中, 如果煤矿有其他工作地点 则需要重点进行标注, 当工作人员参考绘图时能够注意上面 的点内容, 减少其他问题的产生, 而且还能有效提高其数据 获取的准确度。

\section{5 提高煤矿测量技术监督管理工作}

加强对矿产工作的监控和管制, 第一需要做的就是创建 规范的有关管控计划和对于矿产的测绘技能规范, 创建有关 的检查管控部门, 及时对矿产测绘活动实施监察, 计划性地 对测绘工作实施监察。测绘工作者的职业素养对测绘活动的 顺利进行起着至关重要的影响, 尤其是在矿产繁杂的地势、 地质环境中, 测绘工作者需要拥有不怕吃苦、踏实负责、优 良的技术水准等职业素养, 从而确保测绘活动的安全、有效、 精确的实施。所以, 测绘工作者在进行工作以前需要对其实 施有关 的专业技能训练, 然后进行资质考核。另外, 矿产公 司自身应该意识到测绘活动的重要意义, 其是确保矿产 生产 安全与提升矿产经济收益不能缺少的一份子。总的来说, 在 完善和加强矿产测绘技能的监察管控体制的基础之上, 提高 工作者的素养对矿产的测绘工作与矿产行业的安全生产与良 性发展所起到的用处是非常高的。

\section{5 煤矿地质测量技术的改革}

\section{1 测量仪器的更新}

发达国家的煤矿地质测量起步早, 发展历史长, 在长期 的发展之中, 发达国家已经建立了一套完善的煤矿地质测量 体系, 掌握了成熟、先进的煤矿地质测量技术。与其他国家 相比较, 中国煤矿地质测量的起步晚、技术落后、设备版本 滞后老旧等, 在平时的煤矿开采作业之中, 这些因素不能被 忽略, 要重点解决。煤矿作为中国有色矿开采的一份子, 在 以后的煤矿地质开采志宏, 其技术难题需要长时间的解决,
测量仪器和测量技术地不断发展, 使得这一难题得到了有效 地解决, 推动了煤矿地质开采的安全运作。在整体方面, 该 测量工作与其他工程测量有着极高的一致性, 普遍的测量依 据是一定要遵守的。但是, 它的内涵相较其他测量工作来说, 比较丰富, 内容比较纷繁复杂, 那么普遍的测量依据就不再 适用, 它的实施就必须依靠其独特的规范。与以往不同的是, 现在中国的煤矿地质测量仪器正朝着智能化、数字化、小型 便利化的法向发展, 时代在进步, 社会在发展, 大型、笨重 的仪器设备注定会被一步步地淘汰掉, 人工智能、多功能、 全自动的仪器设备是众望所归, 终将被社会所接纳, 但是由 于其价格昂贵, 所以还有一段很长的道路需要走, 中国还无 法普及。随着水准仪和全站仪的全自动化的实现，工作时间 大大缩短, 工作效率得到提升, 省时省力, 同时精准度还可 以保障, 这都是煤矿地质安全生产的重要保障。

\section{2 测量技术的改革}

煤矿煤矿地质开采时离不开测量技术的支持, 测量精准 与否直接关系到煤矿地质生产是否足够安全, 所以这就需要 人们不断地提高测量技术，对其进行换代升级，适应社会发 展的需要, 更安全地保障煤矿地质开采工作安全进行。以前 在进行煤矿地质测量时, 测量人员的测量经验就显得尤其重 要, 因为其作为测量技术的主要依据, 而随着社会的发展, 以往的经验式测量愈发地显得老旧, 已经跟不上时代的发展, 不能再为当今煤矿地质开采提供技术支持了, 不能完成 现在 的测量任务了, 但是也不是说传统技术已经”一无是处”了, 从而将其全盘否定, 全面抛弃, 人们可以取长补短, 将其和 新技术合理地结合，进一步地提升其测量的准确性。当前的 测量技术是数字化测量, 利用数字标记煤矿地质区域的目标, 完成对巷道变形程度的精确计算, 做到实时监测巷道的情况, 降低事故发生的频率, 更能有效地防止事故的发生。煤矿地 质测量技术历时时间较短, 这就导致其测量技术体系不是那 么的完善, 在面对新的测量技术时, 其不能被更新和使用, 为了测量技术可以得到长足的发展, 煤矿地质生产得以安全 展开, 这就要求人们时刻关注技术的发展, 不断地发展、创新, 最终将其更好地应用于煤矿地质生产之中。

\section{6 结语}

随着近年现代化建设的进展, 社会生产对煤炭资源的需 
求仍然很大, 对安全生产的重视也越来越强, 保证煤炭生产 的同时, 还需要保证煤矿生产的安全。煤矿地质测量是地下 开采工程的基本内容之提高准确性对于保障地下工程的安全 具有重要意义。因此, 煤矿地下生产要重视地质测量工作, 加强煤矿测量工作的研究, 矿山实际上要投人先进的技术设 备和专业人才, 最大限度地提高测量精度, 实现对测量精度 的有效控制和优化，从而保证煤矿生产的安全。

\section{参考文献}

[1] 田丰. 地质测量工作的价值与重要性 [J]. 中国石油和化工标准与 质量, 2019(15):49-50.

[2] 郭琳凯. 煤矿地质灾害特征及防治措施研究 [J]. 内蒙古石油化 工 ,2019(07):28-29.

[3] 程志腾. 煤矿地质测量在矿井安全生产中的重要性分析 [J]. 石化 技术 ,2019(07):276-277.

[4] 郭庆换. 煤矿地质钻探中的关键问题 [J]. 当代化工研究, 2019 (06):35-36.
[5] 胡锁云. 煤矿地质勘探技术及其重要性研究 [J]. 中国石油和化工 标准与质量 ,2019 (11):211-212.

[6] 古庆华. 提高煤矿测量精度的对策分析 [J]. 技术与市场, 2019 (3):119-120.

[7] 刘江斌. 煤矿测量方法及提高测量精度的对策探讨 [J]. 中小企业 管理与科技 (下旬刊),2018(12):145-146.

[8] 袁刚. 煤矿地质测量精度提升措施探析 [J]. 内蒙古煤炭经 济,2018(04) :11-12.

[9] 王进. 煤矿测量中提高精度测量措施研究 [J]. 机电工程技 术, 2018(02):36-38.

[10] 王伟. 煤矿测量方法及提高测量精度的对策研究 [J]. 能源与节 能,2018(04):14-15.

[11] 元民. 试析煤矿地质测量中数字化制图技术的应用 [J]. 山东工业 技术, 2018(02):5.

[12] 张华. 地质测量在煤矿安全生产中的作用及对策研究 [J]. 山东煤 炭科技, 2017(03):145-146+149. 